\title{
The effects of tire dynamics on the performance of finite spectrum assignment of vehicle motion control
}

\author{
Illés Vörös ${ }^{1}$, Balázs Várszegi ${ }^{1}$, and Dénes Takács ${ }^{1}$
}

\begin{abstract}
The lateral position control of the vehicle is analyzed in the presence of time delay. In order to compensate the negative effects of deadtime, the predictor control approach called finite spectrum assignment (FSA) is applied. This controller includes a linear model of the plant and uses the solution of this model over the delay interval to predict the current system states. The focus of the paper is whether to include tire dynamics in the predictive model of the controller. Although the more detailed model should improve control performance, the additional parameters (e.g. tire stiffnesses, yaw moment of inertia) are difficult to determine accurately. The effects of parameter mismatches are analyzed in detail and recommendations are given to ensure safe control of the vehicle. It is shown that the inclusion of tire dynamics in the predictive model vastly improves control performance even in the presence of large parameter errors, but in certain cases the inaccuracies may lead to instability.
\end{abstract}

\section{Keywords}

Vehicle dynamics, steering control, time delay, predictor feedback, finite spectrum assignment, lane keeping

\section{Introduction}

According to a recent study by Olofsson and Nielsen (2020), a large percentage of severe lane departure road accidents could have been prevented or their severity greatly reduced with the help of autonomous driving functions. However, due to the increasing complexity of sensor systems, localization and object detection solutions required for safe autonomous driving, time delay has become a serious bottleneck in this field. Our paper aims to highlight the potential benefits of applying predictor feedback in vehicle motion control, directly increasing the safety and efficiency of the related driver assistance and autonomous driving functions.

Many effective control approaches have been proposed over the years for reliable and safe execution of planned trajectories of autonomous vehicles (Paden et al. (2016); Amer et al. (2017)). The most widely used controllers include simple kinematic models, such as pure pursuit (Elbanhawi et al. (2018)) or the Stanley-controller (Hoffmann et al. (2007)). For applications where basic geometric/kinematic considerations might not be sufficient, the dynamic properties of the vehicle can also be included in the controller (Fierro and Lewis (1997)). Advances in computational power have also made the real-time use of model predictive control (MPC) (Beal and Gerdes (2012)) feasible in autonomous vehicles. The easy implementation of higher fidelity models makes MPC also suitable for emergency maneuvers. For a more detailed overview of path following techniques for autonomous vehicles, the reader is referred to Paden et al. (2016) and Amer et al. (2017), and the references therein.

An important aspect of vehicle motion control, which is rarely treated explicitly, is the negative effects of time delay on these control techniques. Several components of the feedback loop contribute to the overall time delay in the system, which adds up to be rather significant (Heredia and Ollero (2007); Kyrychko and Hogan (2010)). The main sources of time delay include sensor and communication delays, signal processing, filtering and actuator dynamics. Algorithms related to perception and localization may also induce significant amounts of delay, especially if visionbased solutions are used (Pendleton et al. (2017)). Although better hardware and more efficient algorithms are introduced day by day, most commercial solutions are still not capable of processing the large amounts of sensor data at a high frequency and it is difficult to find a balance between accuracy, processing time and hardware costs (Liu et al. $(2018,2017))$.

In the following, a few representative examples of numerical time delay values are listed from the literature, in order to give the reader a general sense of what orders of magnitude to expect. In Jalali et al. (2017), a sensor delay of $0.2 \mathrm{~s}$ is considered for yaw rate control. The low level steering angle controller in Mobus and Zomotor (2005) had a time constant of $0.3 \mathrm{~s}$, while Hoffmann et al. (2007) considered $0.4 \mathrm{~s}$ as steering delay. According to Petrovskaya and Thrun (2009), reliable object detection requires 3 frames, which adds up to $0.1 \mathrm{~s}$ with a frame rate of $30 \mathrm{~Hz}$. In Oliveira et al. (2016), an efficient deep learning based algorithm is presented for road surface detection, with a processing time

${ }^{1}$ Department of Applied Mechanics, Budapest University of Technology and Economics, Hungary

\section{Corresponding author:}

Illés Vörös, Department of Applied Mechanics, Budapest University of Technology and Economics, Hungary.

Email: illes.voros@mm.bme.hu 
of $0.05 \mathrm{~s}$. As the delays from various sources start to add up, their negative effects become increasingly difficult to handle.

A possible solution for compensating time delay in the control loop is the use of predictor feedback. Traditionally, predictor control was most commonly used for the control of slower processes with larger time constants, such as in chemical plants. However, due to the increase in available processing power, it is becoming a viable alternative for the automotive industry, too. Based on a mathematical model of the plant, these controllers calculate a prediction of the current system states and use these predictions as feedback instead of the actual, delayed signals. In this paper, the predictor control method called finite spectrum assignment (FSA) is considered (Manitius and Olbrot (1979); Wang et al. (1999); Molnár and Insperger (2016)). In the ideal case, when the predictive model is perfectly accurate, there are no implementation issues and measurement noise in the system, then the predicted states of the FSA controller are equal to the actual system states and the time delay is canceled out.

The performance of the FSA controller was already demonstrated in Vörös and Várszegi (2018), where a simple, kinematic vehicle model was used both for the predictive model in the controller and for the controlled system. In Vörös et al. (2019), the model of the controlled vehicle was extended with tire dynamics, but the internal model remained the same. In the present paper, tire dynamics are included in the predictive model as well. On the one hand, the more precise model should help increase control performance. On the other hand, the additional parameters (cornering stiffnesses, vehicle inertia, etc.) are hard to determine accurately. Therefore it is possible that due to parameter mismatches the more detailed system model might actually perform worse than the simple model with accurate parameters. It will be shown that the presence of parameter mismatches greatly affects the linear stability of the lateral motion, which could even lead to dangerous situations in extreme cases.

The paper is organized as follows: in the first section, two versions of the single track vehicle model are introduced, with and without considering tire dynamics. Next, the stability analysis of delayed state feedback without predictor control is performed as reference. In the following section, two versions of the FSA control law are derived, using the two different vehicle models for prediction. The controllers are then compared based on stability charts. The effects of vehicle cornering characteristics, implementation issues in the control law and robustness against parameter estimation errors are also considered. The results are verified by numerical simulations in the final section.

\section{Vehicle models}

\section{Kinematic vehicle model}

Two vehicle models are considered in this paper: a kinematic and a dynamic version of the single track vehicle model. In the kinematic model (Fig. 1 (a)), point contacts are assumed between the tires and the ground, i.e. no side slip occurs. This can be expressed using kinematic constraints describing the direction of the velocity vectors at the front and rear wheels (points F and R). As a third kinematic constraint, the longitudinal speed of the vehicle is fixed at a constant

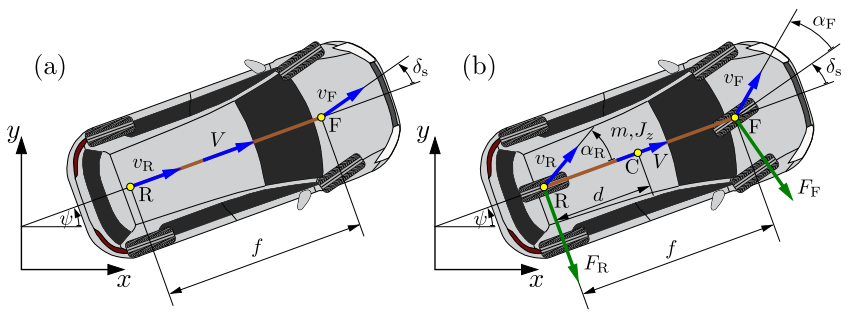

Figure 1. The kinematic vehicle model (a) assuming point contact at the wheels, and the dynamic model (b) including tire side forces.

value $V$. The position of the vehicle is given by the positional coordinates $x$ and $y$ of point $\mathrm{R}$, and by the yaw angle $\psi$. The steering angle is denoted by $\delta_{\mathrm{s}}$. The governing equations of the model can be directly expressed from the three constraint equations (see Vörös and Várszegi (2018) for more details), leading to the system equations:

$\dot{x}(t)=V \cos \psi(t), \dot{y}(t)=V \sin \psi(t), \dot{\psi}(t)=\frac{V}{f} \tan \delta_{\mathrm{s}}(t)$,

where $f$ denotes the vehicle wheelbase.

\section{Dynamic vehicle model}

In the dynamic vehicle model (Fig. 1 (b)), tire side forces are also considered: the lateral forces are assumed to be proportional to the slip angles $\alpha_{i}$ through the cornering stiffnesses $C_{i}$ :

$$
F_{i}^{\mathrm{lat}}=C_{i} \alpha_{i}, \quad i \in\{\mathrm{F}, \mathrm{R}\},
$$

where the indeces $\mathrm{F}$ and $\mathrm{R}$ stand for the front and rear axles respectively. The slip angles are calculated as follows:

$$
\begin{aligned}
\alpha_{\mathrm{F}} & =\arctan \left(\frac{\dot{y}+f \dot{\psi} \cos \psi}{\dot{x}-f \dot{\psi} \sin \psi}\right)-\psi-\delta_{\mathrm{s}}, \\
\alpha_{\mathrm{R}} & =\arctan \left(\frac{\dot{y}}{\dot{x}}\right)-\psi,
\end{aligned}
$$

while the tire side forces in the global (ground-fixed) coordinate system are defined as

$$
\begin{aligned}
& \mathbf{F}_{\mathrm{F}}=\left[\begin{array}{c}
F_{\mathrm{F}, x} \\
F_{\mathrm{F}, y} \\
0
\end{array}\right]=\left[\begin{array}{c}
F_{\mathrm{F}}^{\mathrm{lat}} \sin \left(\psi+\delta_{\mathrm{s}}\right) \\
-F_{\mathrm{F}}^{\mathrm{lat}} \cos \left(\psi+\delta_{\mathrm{s}}\right) \\
0
\end{array}\right], \\
& \mathbf{F}_{\mathrm{R}}=\left[\begin{array}{c}
F_{\mathrm{R}, x} \\
F_{\mathrm{R}, y} \\
0
\end{array}\right]=\left[\begin{array}{c}
F_{\mathrm{R}}^{\mathrm{lat}} \sin \psi \\
-F_{\mathrm{R}}^{\mathrm{lat}} \cos \psi \\
0
\end{array}\right] .
\end{aligned}
$$

As non-zero side slip angles mean that the direction of the velocity vectors are no longer constrained at the wheels, only one kinematic constraint remains, which keeps the longitudinal velocity constant. Since the difference between the number of generalized coordinates (three: $x, y, \psi$ ) and the number of kinematic constraints (one) is two, the velocity state of the model can be described by two variables. When deriving the equations of motion by means of the Gibbs-Appell approach (Greenwood (2006)), two so-called pseudovelocities are defined to realize this description. The pseudovelocities can be chosen intuitively, but they need to 
satisfy the kinematic constraints. We use the lateral speed at point $\mathrm{R}$, denoted by $\sigma_{1}$ and the yaw rate $\sigma_{2}$. The equation of the kinematic constraint along with the definitions of the two pseudovelocities can be solved for the state derivatives:

$$
\begin{aligned}
& \dot{x}=V \cos \psi-\sigma_{1} \sin \psi, \\
& \dot{y}=V \sin \psi+\sigma_{1} \cos \psi, \\
& \dot{\psi}=\sigma_{2} .
\end{aligned}
$$

The remaining two governing equations can be derived from the Gibbs-Appell equations, resulting in

$$
\begin{gathered}
m\left(\dot{\sigma}_{1}-V \sigma_{2}\right)=-\frac{J_{z}+m d^{2}}{J_{z}}\left(\left(F_{\mathrm{F}, x}+F_{\mathrm{R}, x}\right) \sin \psi\right. \\
\left.-\left(F_{\mathrm{F}, y}+F_{\mathrm{R}, y}\right) \cos \psi\right)+\frac{m d f}{J_{z}}\left(F_{\mathrm{F}, x} \sin \psi-F_{\mathrm{F}, y} \cos \psi\right) \\
J_{z} \dot{\sigma}_{2}=\left(F_{\mathrm{F}, x}(d-f)+F_{\mathrm{R}, x} d\right) \sin \psi \\
-\left(F_{\mathrm{F}, y}(d-f)+F_{\mathrm{R}, y} d\right) \cos \psi
\end{gathered}
$$

where the tire force components can be substituted according to (5). The additional vehicle parameters appearing in the dynamical model are the vehicle mass $m$, yaw moment of inertia $J_{z}$ (with respect to the center of gravity) and the distance $d$ between the rear axle and the center of gravity. For the full, detailed derivation of the equations of motion using the Gibbs-Appell method, please refer to Vörös et al. (2019).

\section{Delayed state feedback}

Position control of the dynamic vehicle model in the lateral direction is going to be performed by the proportional feedback of the vehicle's lateral position $y$ and its yaw angle $\psi$. In our study, the steering angle $\delta_{\mathrm{s}}$ is directly generated by the feedback control. Moreover, for the sake of simplicity, we are only considering a straight-line reference trajectory along the $x$-axis, which corresponds to $y=0$ and $\psi=0$. Consequently, the control input can be defined as follows:

$$
\delta_{\mathrm{S}}(t)=-P_{y} y(t-\tau)-P_{\psi} \psi(t-\tau),
$$

where the control gains are denoted by $P_{y}$ and $P_{\psi}$. The delay term $\tau$ represents the total time delay in the system, including sensor delay, communication delays, processing time for all the required algorithms (image processing, position estimation, control, etc.), actuator delay and the dynamics of the steering mechanism. A commonly used, alternative way to consider the time delay in the model is to use a first order lag term with time constant $\tau$, as in e.g. Della Rossa and Mastinu (2018). That way the corresponding delay differential equation is replaced by an ordinary differential equation, which is easier to handle mathematically.

After linearizing the dynamic vehicle model with control law (9) around the equilibrium state of rectilinear motion along the $x$-axis, the following state-space representation can be applied:

$$
\dot{\mathbf{x}}(t)=\mathbf{A x}(t)+\mathbf{B u}(t-\tau),
$$

where the single control input is the steering angle: $\mathbf{u}(t)=$ $-P_{y} y(t)-P_{\psi} \psi(t)$. Since the longitudinal position of the vehicle does not influence stability, the corresponding equation can be decoupled. Therefore, in the rest of the paper, we are only going to consider the reduced state vector $\mathbf{x}=\left[\begin{array}{llll}y & \psi & \sigma_{1} & \sigma_{2}\end{array}\right]^{\mathrm{T}}$. The corresponding system and input matrices are

$$
\mathbf{A}=\left[\begin{array}{cccc}
0 & V & 1 & 0 \\
0 & 0 & 0 & 1 \\
0 & 0 & A_{33} & A_{34} \\
0 & 0 & A_{43} & A_{44}
\end{array}\right], \quad \mathbf{B}=\left[\begin{array}{c}
0 \\
0 \\
B_{3} \\
B_{4}
\end{array}\right],
$$

with elements

$$
\begin{gathered}
A_{33}=-\frac{B_{3}}{V}-\frac{C_{\mathrm{R}}\left(J_{z}+m d^{2}\right)}{m V J_{z}}, \quad A_{34}=-B_{3} \frac{f}{V}-V, \\
A_{43}=-\frac{B_{4}}{V}+\frac{C_{\mathrm{R}} d}{V J_{z}}, \quad A_{44}=-B_{4} \frac{f}{V},
\end{gathered}
$$

and

$$
B_{3}=\frac{C_{\mathrm{F}}\left(J_{z}+m d(d-f)\right)}{m J_{z}}, \quad B_{4}=\frac{C_{\mathrm{F}}(f-d)}{J_{z}} .
$$

Using direct state feedback, the steering angle can be written as $\mathbf{u}(t-\tau)=\mathbf{K} \mathbf{x}(t-\tau)$, where $\mathbf{K}=\left[\begin{array}{llll}-P_{y} & -P_{\psi} & 0 & 0\end{array}\right]$ includes the control gains. This leads to the characteristic equation

$$
D(\lambda):=\operatorname{det}\left(\lambda \mathbf{I}-\mathbf{A}-\mathbf{B K} \mathrm{e}^{-\lambda \tau}\right)=0,
$$

where $\lambda \in \mathbb{C}$ is the characteristic exponent and $\mathbf{I}$ denotes the identity matrix.

The stability analysis of the system with feedback delay can be performed using the D-subdivision method. When $\lambda=0$, the system loses its stability without oscillations (static loss of stability), which translates into the stability boundary $P_{y}=0$. Furthermore, at the boundaries of dynamic loss of stability, the characteristic exponent has no real part, i.e. $\lambda=\mathrm{i} \omega$. Substituting this into (14), then separating the real and imaginary parts of the equation, $P_{y}$ and $P_{\psi}$ can be expressed as a function of $\omega$ ( $\omega$ represents the angular frequency of the arising oscillations). These parametric expressions can be used to plot the so-called D-curves that separate domains of the $\left(P_{y}, P_{\psi}\right)$ plane according to the number of unstable characteristic exponents. Control gains from the region where the number of unstable characteristic roots is zero are going to lead to stable system behavior. To determine this number at a given point of the stability map, Stepan's formulae (Stépán (1989)) can be used.

The stability chart is plotted in Fig. 2 (a) for different time delays. When there is no delay in the system, the stable domain is unbounded, otherwise it rapidly shrinks with increasing time delay. The stability analysis of the dynamic vehicle model using the control law (9) is presented in more detail in Vörös et al. (2019).

\section{Finite spectrum assignment}

Similarly to the classical Smith predictor, finite spectrum assignment compensates time delay by predicting the actual, delay-free system states by solving a mathematical model of 
the plant (Manitius and Olbrot (1979); Wang et al. (1999)). This solution is then used as feedback, instead of the delayed measurement or observer signals. First, a summary of the theoretical background of the FSA controller is going to be presented, then we are going to apply it to the dynamic vehicle model.

\section{Theoretical background}

In order to predict the current state of system (10), the FSA controller employs an internal model

$$
\dot{\mathbf{x}}(t)=\widetilde{\mathbf{A}} \mathbf{x}(t)+\widetilde{\mathbf{B}} \mathbf{u}(t-\tilde{\tau}),
$$

where tildes are used to differentiate the model parameters used within the controller from their real values. The predictor solves (15) over the assumed delay interval $\tilde{\tau}$ by taking the latest available measurement as initial condition. This results in the predicted state

$$
\mathbf{x}_{\mathrm{p}}(t+\tilde{\tau})=\mathrm{e}^{\widetilde{\mathbf{A}} \tilde{\tau}} \mathbf{x}(t)+\int_{-\tilde{\tau}}^{0} \mathrm{e}^{-\widetilde{\mathbf{A}} s} \widetilde{\mathbf{B}} \mathbf{u}(t+s) \mathrm{d} s,
$$

which is then used as feedback in the control law:

$$
\mathbf{u}(t)=\mathbf{K} \mathrm{e}^{\widetilde{\mathbf{A}} \tilde{\tau}} \mathbf{x}(t)+\mathbf{K} \int_{-\tilde{\tau}}^{0} \mathrm{e}^{-\widetilde{\mathbf{A}} s} \widetilde{\mathbf{B}} \mathbf{u}(t+s) \mathrm{d} s
$$

If the internal model perfectly matches the real system $(\widetilde{\mathbf{A}} \equiv$ $\mathbf{A}, \widetilde{\mathbf{B}} \equiv \mathbf{B}$ and $\tilde{\tau} \equiv \tau)$, then the control law (17) leads to the closed loop dynamics $\dot{\mathbf{x}}(t)=(\mathbf{A}+\mathbf{B K}) \mathbf{x}(t)$, which is the same as simple delay-free state feedback. Thus the FSA controller is capable of removing the effects of time delay from the feedback loop, reducing the infinite dimensional spectrum of the original problem to a finite number of poles. If the pair $\mathbf{A}$ and $\mathbf{B}$ is controllable, then the remaining poles can be freely assigned through the feedback matrix $\mathbf{K}$, achieving stability for arbitrarily large values of $\tau$.

However, this ideal implementation of FSA is only possible in theory. On the one hand, there is always some degree of uncertainty in the model parameters $\widetilde{\mathbf{A}}, \widetilde{\mathbf{B}}$ and $\tilde{\tau}$, as well as unmodeled dynamics, non-linearities and noise that are not considered in the system model. Therefore the predictive model will always differ from the real system and the delayed terms cannot be canceled out. As a result, the system's spectrum will remain infinite dimensional and the poles cannot be assigned freely.

On the other hand, the implementation of the integral part of (17) is not trivial. Approximating it with numerical quadrature leads to

$$
\mathbf{u}(t)=\mathbf{K} \mathrm{e}^{\widetilde{\mathbf{A}} \tilde{\tau}} \mathbf{x}(t)+\mathbf{K} \sum_{j=0}^{\tilde{r}} \mathrm{e}^{\widetilde{\mathbf{A}} \theta_{j, \tilde{r}}} \widetilde{\mathbf{B}} \mathbf{u}\left(t-\theta_{j, \tilde{r}}\right) h_{j, \tilde{r}}
$$

where $\theta_{j, \tilde{r}} \in[0, \tilde{\tau}], \quad h_{j, \tilde{r}} \in \mathbb{R}, \quad$ and the integer $\tilde{r} \in \mathbb{Z}^{+}$ determines the accuracy of the approximation: as $\tilde{r} \rightarrow \infty$ the summation tends to the exact value of the integral. This approximation, however, qualitatively changes the nature of the system, resulting in a set of neutral functional differential equations:

$$
\begin{aligned}
\dot{\mathbf{x}}(t)= & \mathbf{A x}(t)+\mathbf{B} \mathbf{u}(t-\tau), \\
\dot{\mathbf{u}}(t)= & \mathbf{K} \mathrm{e}^{\widetilde{\mathbf{A}} \tilde{\tau}} \mathbf{A} \mathbf{x}(t)+\mathbf{K} \mathrm{e}^{\widetilde{\mathbf{A}} \tilde{\tau}} \mathbf{B u}(t-\tau) \\
& +\sum_{j=0}^{\tilde{r}} \mathbf{K} \mathrm{e}^{\widetilde{\mathbf{A}} \theta_{j, \tilde{r}}} \widetilde{\mathbf{B}} \dot{\mathbf{u}}\left(t-\theta_{j, \tilde{r}}\right) h_{j, \tilde{r}} .
\end{aligned}
$$

It can be shown that the replacement of (17) with (18) leads to the appearance of a set of additional characteristic exponents called the essential spectrum (Mondié et al. (2002); Michiels and Niculescu (2007); Michiels et al. (2003)). As $\tilde{r} \rightarrow \infty$, the imaginary parts of these roots move off to infinity, but their real parts may have a finite, positive limit, leading to stability issues regardless of the accuracy of the approximation. In order to guarantee stability of the closed loop system with control law (18), the stability of the difference part of (19) also needs to be ensured (Mondié et al. (2002)):

$$
\begin{aligned}
& \mathbf{x}(t)=\mathbf{0} \\
& \mathbf{u}(t)=\sum_{j=0}^{\tilde{r}} \mathbf{K} \mathrm{e}^{\widetilde{\mathbf{A}} \theta_{j, \tilde{r}}} \widetilde{\mathbf{B}} \mathbf{u}\left(t-\theta_{j, \tilde{r}}\right) h_{j, \tilde{r}}
\end{aligned}
$$

If $\tilde{r}$ is large enough, this condition can be approximated by the stability of

$$
\mathbf{u}(t)=\mathbf{K} \int_{-\tilde{\tau}}^{0} \mathrm{e}^{-\widetilde{\mathbf{A}} s} \widetilde{\mathbf{B}} \mathbf{u}(t+s) \mathrm{d} s .
$$

As the essential spectrum is not continuous in $\theta_{j, \tilde{r}}$ (Michiels and Niculescu (2007); Michiels et al. (2003)), perturbations of the integral time step may also lead to instability. Therefore, stability of the difference part (20) may depend on the choice of integration scheme. In order to guarantee robustness against perturbations of $\theta_{j, \tilde{r}}$, the strong stability of (20) is required (Michiels et al. (2003)). This can be ensured if

$$
S=\int_{0}^{\tilde{\tau}}\left|\mathbf{K} \mathrm{e}^{\widetilde{\mathbf{A}} s} \widetilde{\mathbf{B}}\right| \mathrm{d} s<1 .
$$

Following Michiels and Niculescu (2007), we are going to refer to the stability of the closed loop system with an accurately implemented control law (17) as ideal stability. If the functional differential equation (21) is also stable, it will be referred to as theoretical stability. In addition, if robustness against perturbations of the integral time step is also ensured based on (22), we refer to it as robust stability.

The instability mechanisms of theoretical and robust stability are caused by unstable characteristic roots with large imaginary parts. Therefore these are high frequency phenomena that can be avoided by applying a low-pass filter (Mondié and Michiels (2003)) or by using a digital controller (Michiels and Niculescu (2007)).

\section{FSA based on the kinematic vehicle model}

In order to apply the FSA controller to the vehicle position control problem, we are going to include the two previously detailed vehicle models within the controller. First, the 
linearized kinematic vehicle model is of the form

$$
\left[\begin{array}{c}
\dot{y}(t) \\
\dot{\psi}(t)
\end{array}\right]=\underbrace{\left[\begin{array}{cc}
0 & \tilde{V} \\
0 & 0
\end{array}\right]}_{\widetilde{\mathbf{A}}_{\mathrm{kin}}}\left[\begin{array}{c}
y(t) \\
\psi(t)
\end{array}\right]+\underbrace{\left[\begin{array}{c}
0 \\
\tilde{V} / \tilde{f}
\end{array}\right]}_{\widetilde{\mathbf{B}}_{\mathrm{kin}}} \mathbf{u}(t-\tilde{\tau}),
$$

where tildes denote the model parameters used within the controller. Using this model for predictions, the steering angle according to (17) is generated as follows:

$$
\begin{aligned}
\mathbf{u}(t)= & {\left[\begin{array}{ll}
-P_{y} & -P_{\psi}
\end{array}\right]\left(\left[\begin{array}{cc}
1 & \tilde{V} \tilde{\tau} \\
0 & 1
\end{array}\right]\left[\begin{array}{c}
y(t) \\
\psi(t)
\end{array}\right]\right.} \\
& \left.+\int_{-\tilde{\tau}}^{0}\left[\begin{array}{cc}
1 & -s \tilde{V} \\
0 & 1
\end{array}\right]\left[\begin{array}{c}
0 \\
\tilde{V} / \tilde{f}
\end{array}\right] \mathbf{u}(t+s) \mathrm{d} s\right) .
\end{aligned}
$$

The solution of the system consisting of the linearized dynamic vehicle model (10) and the control law (24) is assumed to be in the form $\mathbf{C e}^{\lambda t}\left(\mathbf{C} \in \mathbb{C}^{5}\right)$. Substituting this trial function leads to the system of equations

$$
\underbrace{\left[\begin{array}{ccccc}
\lambda & -V & -1 & 0 & 0 \\
0 & \lambda & 0 & -1 & 0 \\
0 & 0 & \lambda-A_{33} & -A_{34} & -B_{3} \mathrm{e}^{-\lambda \tau} \\
0 & 0 & -A_{43} & \lambda-A_{44} & -B_{4} \mathrm{e}^{-\lambda \tau} \\
P_{y} & P_{\psi}+P_{y} \tilde{V} \tilde{\tau} & 0 & 0 & g(\lambda)
\end{array}\right]}_{\mathbf{M}_{\mathrm{kin}}(\lambda)} \mathbf{C}=
$$

where the coefficient matrix $\mathbf{M}_{\text {kin }}(\lambda)$ includes the elements of $\mathbf{A}$ and $\mathbf{B}$ from the system model (11), and the last row corresponds to the control law with

$$
\begin{aligned}
g(\lambda)= & \frac{1}{\tilde{f} \lambda^{2}}\left(\tilde{f} \lambda^{2}-\tilde{V} \mathrm{e}^{-\lambda \tilde{\tau}}\left(P_{y}(\lambda \tilde{\tau} \tilde{V}+\tilde{V})+\lambda P_{\psi}\right)\right. \\
& \left.+P_{y} \tilde{V}^{2}+\lambda P_{\psi} \tilde{V}\right) .
\end{aligned}
$$

Taking the determinant of $\mathbf{M}_{\text {kin }}(\lambda)$ leads to the characteristic equation using the kinematic vehicle model for predictions:

$$
D_{\text {kin }}(\lambda):=\operatorname{det}\left(\mathbf{M}_{\text {kin }}(\lambda)\right)=0 .
$$

\section{FSA based on the dynamic vehicle model}

The linear form of the dynamic vehicle model is shown in equations (10)-(13). The corresponding predictive matrices $\widetilde{\mathbf{A}}_{\mathrm{dyn}}$ and $\widetilde{\mathbf{B}}_{\mathrm{dyn}}$ are the same as in (11), but the parameter values used for prediction are differentiated from their real values with tildes. The FSA control law using the dynamic vehicle model is

$$
\begin{aligned}
\mathbf{u}(t)= & {\left[\begin{array}{c}
-P_{y} \\
-P_{\psi} \\
0 \\
0
\end{array}\right]^{\mathrm{T}}\left(\mathrm{e}^{\widetilde{\mathbf{A}}_{\mathrm{dyn}} \tilde{\tau}}\left[\begin{array}{c}
y(t) \\
\psi(t) \\
\sigma_{1}(t) \\
\sigma_{2}(t)
\end{array}\right]\right.} \\
& \left.+\int_{-\tilde{\tau}}^{0} \mathrm{e}^{-\widetilde{\mathbf{A}}_{\mathrm{dyn}} s} \widetilde{\mathbf{B}}_{\mathrm{dyn}} \mathbf{u}(t+s) \mathrm{d} s\right) .
\end{aligned}
$$

Note that the dynamic vehicle model also provides predictions about the vehicle's lateral velocity and yaw rate. However, in order to directly compare the control performance of the two predictive models, we are not feeding these additional predicted states back, hence the corresponding control gains are set to zero.

Substituting the exponential trial function $\mathbf{C e}^{\lambda t}$, the system consisting of (10) and (28) can be written in matrix form similarly to (25). Since only the control law is changed, the resulting coefficient matrix $\mathbf{M}_{\mathrm{dyn}}(\lambda)$ only differs from $\mathbf{M}_{\mathrm{kin}}(\lambda)$ in its last row. This leads to the characteristic equation

$$
D_{\text {dyn }}(\lambda):=\operatorname{det}\left(\mathbf{M}_{\text {dyn }}(\lambda)\right)=0 .
$$
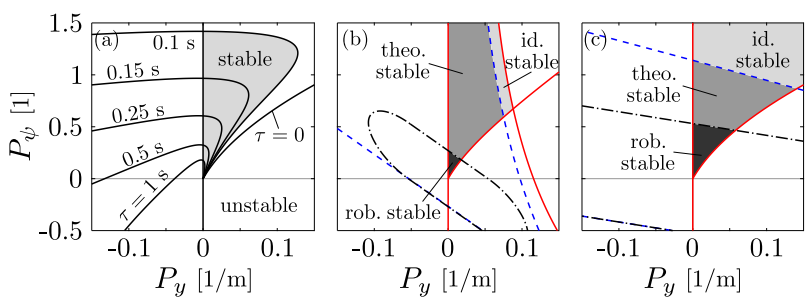

Figure 2. Stability charts of the three investigated controllers: (a) delayed state feedback (the shaded region corresponds to $\tau=0.1 \mathrm{~s}$ ), (b) FSA using the kinematic vehicle model and (c) FSA using the dynamic vehicle model. The time delay considered in the FSA controller is $\tau=0.5 \mathrm{~s}$. Vehicle parameters are listed in Table 1. Zoomed out versions of panel (b) and (c) can be seen in Fig. 3.

\section{Stability analysis}

The characteristic equations $D_{\text {kin }}(\lambda)$ and $D_{\text {dyn }}(\lambda)$ are related to the notion of ideal stability. Performing the Dsubdivision method on them leads to the stability curves shown in Fig. 2 (b) and (c) in red.

For theoretical stability, which is related to the numerical approximation of the FSA control law, the characteristic equation of (21) needs to be analyzed. This can be reached by substituting $\mathbf{x}(t) \equiv \mathbf{0}$ and $\delta_{\mathrm{s}}(t)=\delta_{\mathrm{s}, 0} \mathrm{e}^{\lambda t}$ into the control law. In case of the kinematic FSA this leads to

$$
\begin{aligned}
D_{\text {kin }, \text { th. }}(\lambda)= & \frac{1}{\tilde{f} \lambda^{2}}\left(\left(\lambda\left(\tilde{f} \lambda+P_{\psi} \tilde{V}\right)+P_{y} \tilde{V}^{2}\right)\right. \\
& \left.-\mathrm{e}^{-\lambda \tilde{\tau}} \tilde{V}\left(P_{y}(\lambda \tilde{\tau} \tilde{V}+\tilde{V})+\lambda P_{\psi}\right)\right),
\end{aligned}
$$

while the dynamic model leads to a very long expression that we are not going to include here for the sake of brevity.

Robustness to changes in the integration time step can be ensured based on (22), leading to

$$
S_{\text {kin }}=\int_{0}^{\tilde{\tau}}\left|\frac{\tilde{V}\left(-P_{y} s \tilde{V}-P_{\psi}\right)}{\tilde{f}}\right| \mathrm{d} s<1
$$

for the kinematic model, and the expression

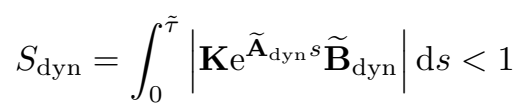

for the dynamic model. The stability boundaries of theoretical and robust stability are plotted in dashed blue and black dash-dot lines in Fig. 2, respectively. Numerical simulations demonstrating the significance of these additional stability regions can be found in Vörös and 
Várszegi (2018); Molnár and Insperger (2016) and in Vörös et al. (2019).

Since all the model parameters are assumed to be known precisely, the region of ideal stability using the dynamic vehicle model is the same as when using simple state feedback with no time delay (see panels (a) and (c) in Fig. 2). When the kinematic model is used for prediction, the delayed terms cannot be canceled out completely, which leads to significantly smaller stable regions (Fig. 2 (b)). Nevertheless, the stable areas of the kinematic FSA are still considerably larger than in the case of delayed state feedback, therefore even though the predictions are not completely accurate, they still provide a great improvement over direct state feedback.

\section{Stability charts}

\section{Effects of vehicle steering characteristics}

In the following, it is shown how the steering characteristics of the vehicle affect the stable parameter domains. In the three columns of Fig. 3, the stability maps are plotted for an oversteering, neutral and understeering vehicle, respectively. The cornering stiffness at the front wheels was fixed at $C_{\mathrm{F}}=45 \mathrm{kN}$, while the different steering characteristics were achieved by varying the rear cornering stiffness as follows: $C_{\mathrm{R}}=15 \mathrm{kN}$ (oversteer), $C_{\mathrm{R}}=45 \mathrm{kN}$ (neutral) and $C_{\mathrm{R}}=$ $75 \mathrm{kN}$ (understeer). The (unrealistically) strong understeer and oversteer parameters were chosen intentionally, in order to accentuate the qualitative changes in the stability maps. The rest of the vehicle parameters are shown in Table 1 and no parameter mismatches were considered in the control law.

An oversteering vehicle design is generally more prone to instability. The vehicle parameters in cases (a) and (d) are chosen such that the critical speed above which steady state cornering is unstable, is exceeded (this was achieved by setting the cornering stiffness at the rear axle unrealistically low). Therefore, the origin of the stability maps, which corresponds to no control action is not part of the stable domain. In case of the kinematic FSA, stable $\left(P_{y}, P_{\psi}\right)$ parameter pairs exist only if the time delay is sufficiently small so that modeling errors do not accumulate too much during the integration. On the other hand, the dynamic FSA can still stabilize the vehicle with appropriately chosen control gains, since it includes information about the cornering behavior. However, as shown in Fig. 3 (d), it is possible that there is no intersection of the ideally stable domain and the theoretically or robustly stable regions.

An understeering vehicle characteristic does not seem to change the ideally stable region much, although it benefits the domains of theoretical and robust stability when using the dynamic model in the controller. Since the kinematic FSA does not include tire parameters, the theoretical and robust stability curves remain the same in all three cases.

\section{Effects of parameter mismatches in case of dynamic FSA controller}

Including tire dynamics in the predictive model is clearly beneficial in terms of stability and performance, since only in this way can the delay completely canceled out from the characteristic equation. However, the dynamic vehicle model needed for this includes a number of additional
Table 1. Vehicle parameters used in stability maps and simulations (unless noted otherwise).

\begin{tabular}{lcc}
\hline Parameter & Notation & Value \\
\hline Vehicle wheelbase & $f$ & $2.7 \mathrm{~m}$ \\
Distance between rear & $d$ & $1.35 \mathrm{~m}$ \\
axle and center of gravity & $m$ & $1430 \mathrm{~kg}$ \\
Vehicle mass & $J_{z}$ & $2500 \mathrm{kgm}$ \\
Yaw moment of inertia & $C_{\mathrm{F}}$ & $45 \mathrm{kN}$ \\
Lateral stiffness of front tire & $C_{\mathrm{R}}$ & $45 \mathrm{kN}$ \\
Lateral stiffness of rear tire & $V$ & $20 \mathrm{~m} / \mathrm{s}$ \\
Longitudinal velocity & $\tau$ & $0.5 \mathrm{~s}$ \\
Time delay & & \\
\hline
\end{tabular}

parameters that are hard to determine accurately (e.g. cornering stiffnesses, moment of inertia). If the parameter values used in the predictive model are inaccurate, delayed terms remain in the characteristic equation, which leads to less than optimal performance and may even cause loss of stability. Therefore it is important to check the robustness of the system against parameter mismatches.

In Fig. 4, the ideally stable domains are plotted for a neutrally steered vehicle with parameters in Table 1, when the front and rear cornering stiffness values in the predictive model are $0 \%, \pm 20 \%$ or $\pm 60 \%$ inaccurate. The cornering stiffness of the tires may change according to the vertical load, inflation pressure, environmental conditions (temperature, etc.), tire wear, or by changing tires on the vehicle (Pacejka (2006)). Moreover, tire related quantities are generally only indirectly estimated based on the dynamics of the whole vehicle, therefore estimation errors are to be expected.

The stability charts in Fig. 4 show that inaccuracies in the cornering stiffness can severely reduce the initially unbounded stable area, especially when very soft tires are assumed. The analysis in the previous section showed that the regions of theoretical and robust stability are concentrated near the origin. Moreover, the optimal control parameters that will be used in the following section are also located close to the origin, therefore this area of the stable domain has the most significance in practice. This region is not affected much by overestimating the tire parameters, but erring to the other side might lead to instability because of the sudden reduction of the stable area. Therefore it is recommended to round uncertainties to the upper side. As the estimated tire stiffness in the dynamic FSA is increased further and further, the stable region tends to the stable domain of the kinematic FSA, which assumes infinitely stiff tires.

It also seems to be safer to assume an oversteering vehicle characteristic instead of an understeering one. This also makes physical sense, since if an understeering cornering behavior is assumed, the controller might overcompensate, leading to instabilities. On the other hand, if the controller thinks that the vehicle is more sensitive than in reality, it will only lead to slower, less aggressive control action.

The effects of inaccuracies in terms of vehicle mass and moment of inertia are shown in Fig. 5. In both cases, the system proves to be more robust against overestimated parameters than underestimates, as long as the errors are not too large. It is also worth noting that the system is clearly more sensitive to uncertainties in terms of moment 

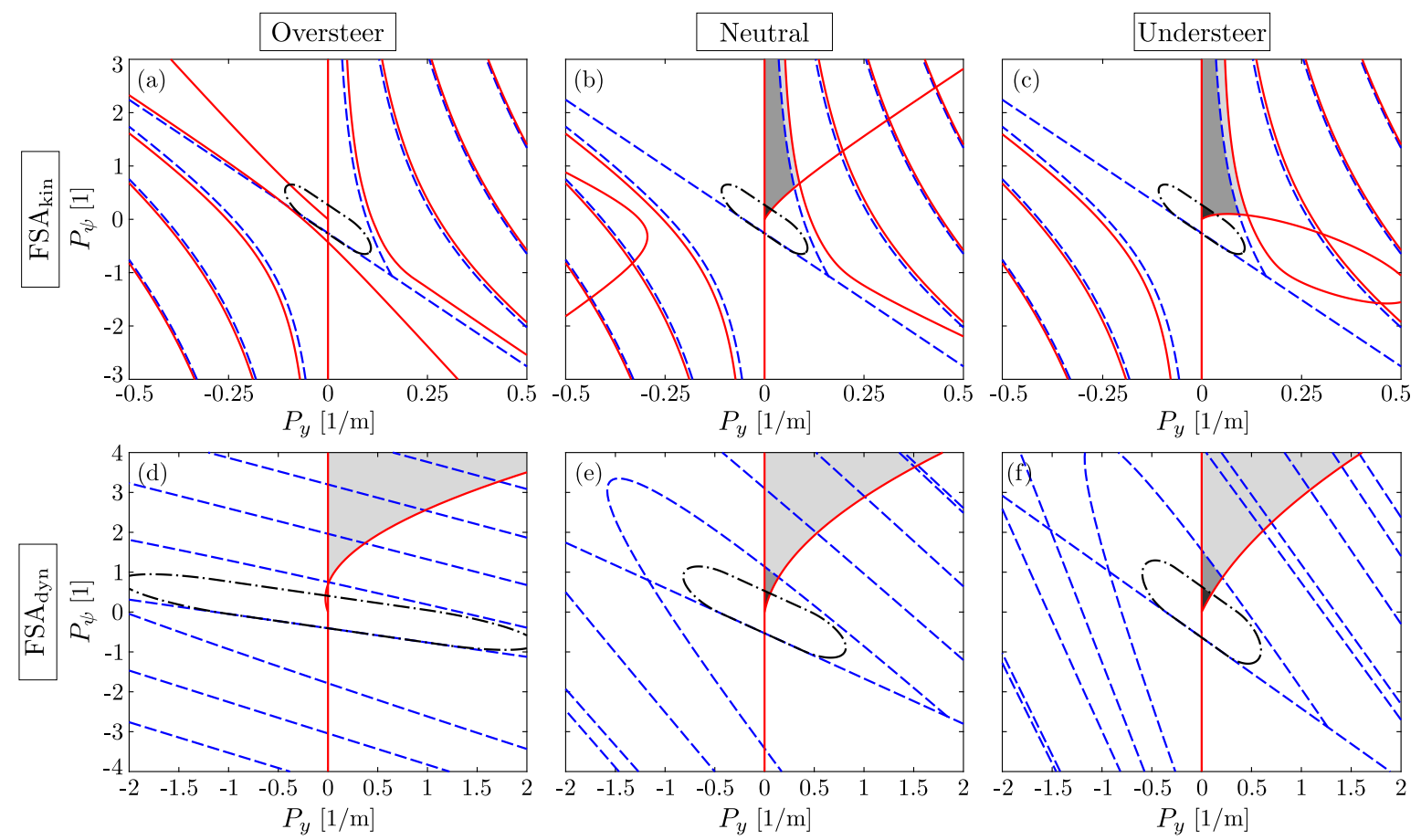

Figure 3. Stability charts of the FSA controller for different steering characteristics using the kinematic (first row) and dynamic (second row) vehicle model for prediction. Solid red lines indicate the stability boundaries of the ideal closed loop system, dashed blue lines the stability boundaries of (21) and black dash-dot lines correspond to the boundary of the robust stability condition (22). The ideally, theoretically and robustly stable domains are shown in light gray, dark gray and black, respectively.

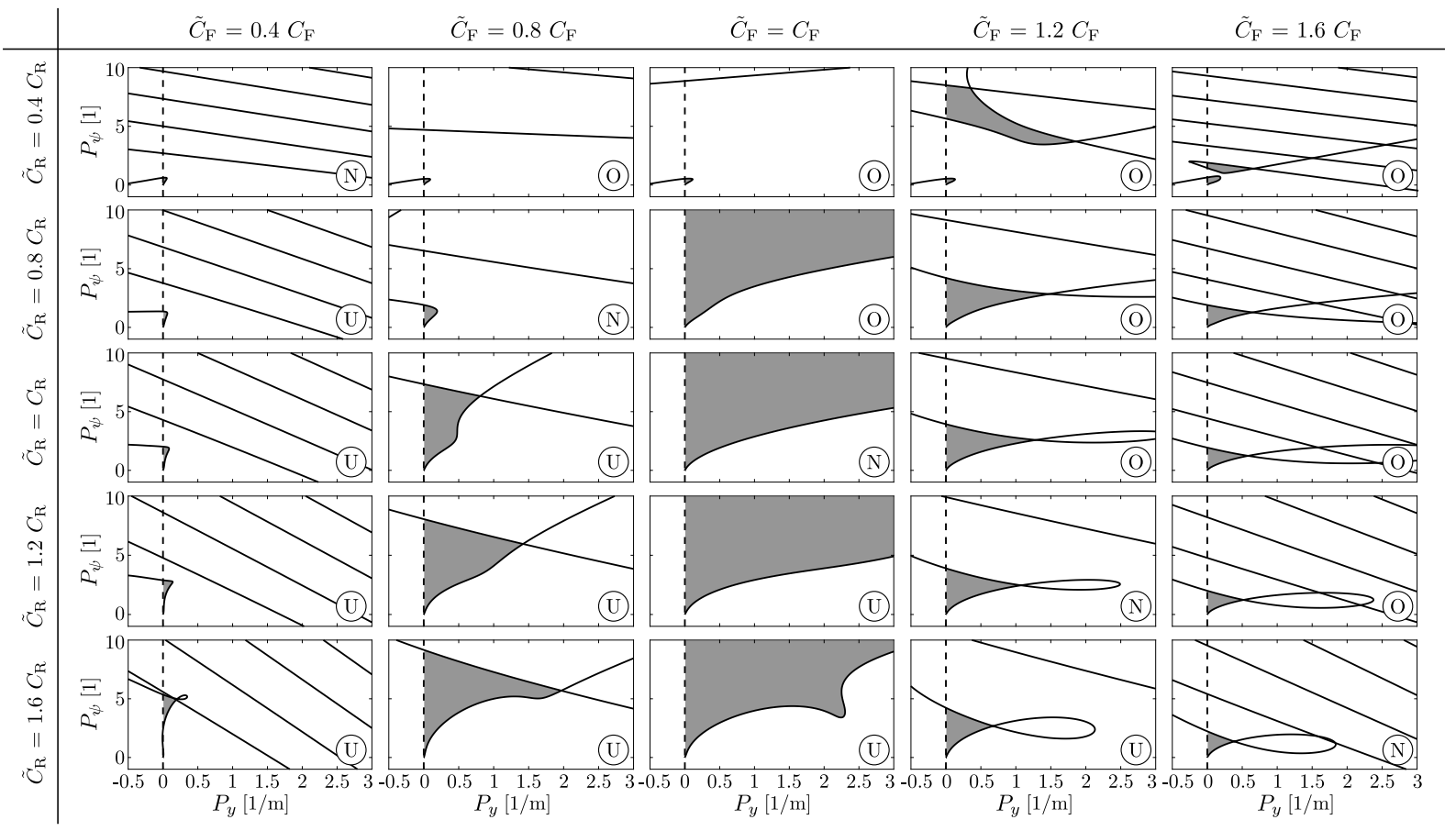

Assumed steering characteristic of the predictive model: (N) neutral, (U) understeer, (O) oversteer

Figure 4. The ideally stable domain of the dynamic FSA controller for $0 \%, \pm 20 \%$ and/or $\pm 60 \%$ inaccuracies in the tire cornering stiffnesses. The stable regions are shown in grey. Vehicle parameters are listed in Table 1.

of inertia than to inaccuracies in the vehicle mass. Even if the estimated mass is half or double the real value, the stable region remains fairly large. On the other hand, $20 \%$ error in $J_{z}$ already greatly reduces the stable area regardless of the direction of the inaccuracy.

\section{Performance analysis}

In order to assess the effects of the previously detailed parameter errors on the actual control performance, a number of numerical simulations were run. We used the non-linear dynamic vehicle model extended with the full, non-linear form of the brush tire model (Pacejka (2006)) for the simulations in order to capture the saturation of the lateral 


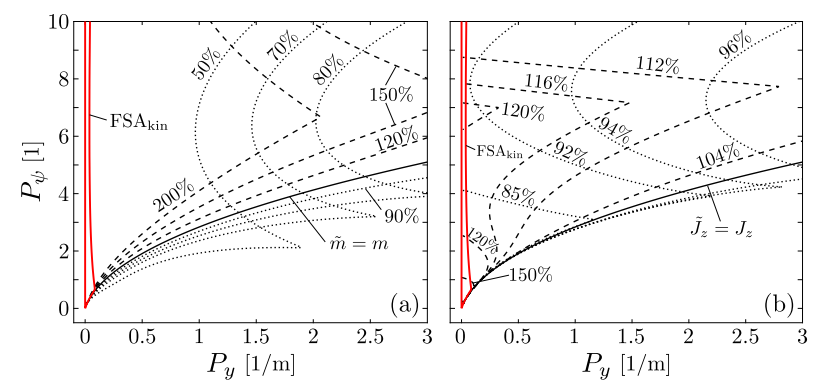

Figure 5. Stability boundaries of the dynamic FSA controller for various amounts of parameter mismatches in terms of (a) vehicle mass and (b) moment of inertia. Underestimated cases are shown in dotted lines $\left(\tilde{m}<m, \tilde{J}_{z}<J\right)$, overestimates in dashed $\left(\tilde{m}>m, \tilde{J}_{z}>J\right)$ and solid lines represent the accurate model $\left(\tilde{m}=m, \tilde{J}_{z}=J\right)$. The boundary of the kinematic FSA is shown in red for reference. Vehicle parameters are listed in Table 1.

forces (the coefficient of friction was set to 0.9). Moreover, the steering angle was limited at $\pm 40^{\circ}$. Although in most cases, the vehicle stayed near the investigated equilibrium, these small additions to the model help make it stay valid in a larger part of the phase space. A higher degree of freedom vehicle model could also be used for the simulations for added accuracy (e.g. see the models in Mashadi et al. (2013) and Mashadi et al. (2014)), but we wanted to make sure that the simulation results are directly comparable to the stability maps. Moreover, the observed lateral accelerations remain low enough so that the vehicle dynamics stay within the limits of validity of the single-track model (Segel (1956)).

The initial conditions were set to model a lane change: $y(0)=3.75 \mathrm{~m}, \psi(0)=0$. For $t \in[-\tau, 0)$, both $y$ and $\psi$ were set to zero, representing that a change in the reference signal (i.e. the decision to change lanes) only occurs at $t=0$. Since the different sources of time delay are not separated in our model, this information only reaches the controllers at $t=\tau$ and no control action is produced until that point. The simulations were run with a timestep of $0.001 \mathrm{~s}$, while the FSA controllers used an integration timestep of $0.05 \mathrm{~s}$. The control gains were chosen to provide the most highly damped system response: first, the system was converted to its semi-discrete form (Insperger and Stépán (2011)), then its characteristic multipliers were evaluated point by point along the stability maps. We chose the control gains where the characteristic multipliers had the smallest modulus. For delayed state feedback these are $P_{y}=0.00077 \mathrm{~m}^{-1}$, $P_{\psi}=0.0805$, for the kinematic FSA $P_{y}=0.0016 \mathrm{~m}^{-1}$, $P_{\psi}=0.1253$ and for the dynamic FSA controller $P_{y}=$ $0.0138 \mathrm{~m}^{-1}, P_{\psi}=0.472$.

Figure 6 shows a comparison of the three controllers in the ideal case, when no parameter mismatches are present. The time delay severely restricts the stable parameter domain of the simple feedback controller, therefore the small control gains lead to a rather slow system response with negligible side slip angles and lateral acceleration $a_{\text {lat }}$. Although using the kinematic vehicle model for predictions allows the use of larger control gains, it only leads to a small improvement in terms of settling time (see Table 2 for numerical values; our definition of settling time was the lowest time instant $t^{*}$ for which $\left.|y(t)|<0.02|y(0)| \forall t>t^{*}\right)$. On the other

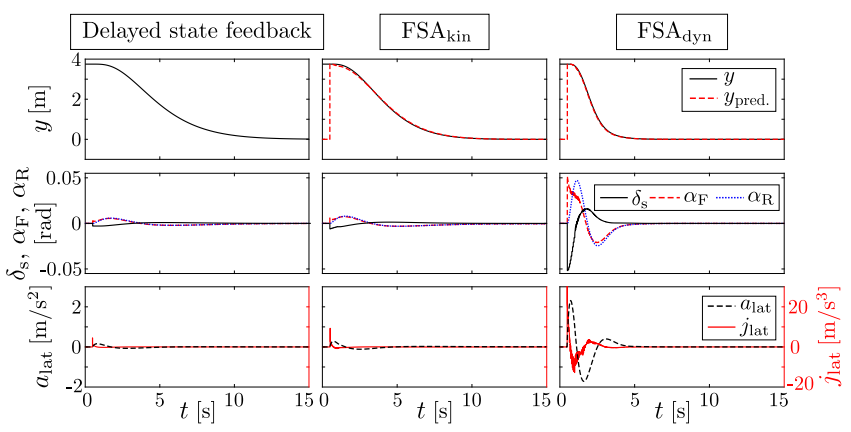

Figure 6. Simulation results of the three controllers with no parameter mismatches in the predictive model of FSA. Vehicle parameters are listed in Table 1 and numerical results in Table 2.

hand, the dynamic FSA can handle even larger control gains, which results in a significantly faster system response. This is achieved through a more aggressive turning of the steering wheel, which also leads to larger side slip angles $\alpha_{\mathrm{F}}, \alpha_{\mathrm{R}}$, lateral acceleration $a_{\text {lat }}$ and jerk $j_{\text {lat }}$. Note that since the reference signal is zero up until $t=0$, the controllers assume zero tracking error during the first $\tau$ interval.

The simulation results in Fig. 7 show that the dynamic FSA outperforms the other two controllers even when the cornering stiffness values are highly uncertain. There is no significant change in the system response as long as the predictive model is tuned to have a neutral or oversteering cornering characteristic. On the other hand, choosing an understeering behavior can lead to undesired oscillations and even instability in extreme cases. As an illustrative example, it is more preferable to overestimate both $C_{\mathrm{F}}$ and $C_{\mathrm{R}}$ by $60 \%$ (leading to a neutral predictive model), than knowing the exact value of $C_{\mathrm{F}}$ and having the $60 \%$ error only in $C_{\mathrm{R}}$. Even though only one parameter is inaccurate, the latter scenario leads to an understeering predictive model, which results in the appearance of oscillations in the system response.

Based on our analysis so far, if the precise value of $C_{\mathrm{F}}$, $C_{\mathrm{R}}, m$ or $J_{z}$ are not known, from a practical point of view, it is generally more beneficial to overestimate them (as long as the predictive model does not become understeered as a result). In Fig. 8, a sort of worst case scenario is considered, where all additional physical parameters of the dynamic vehicle model are highly uncertain: the front and rear cornering stiffnesses are assumed to be double their real value, while $m$ and $J_{z}$ are overestimated by $50 \%$. Despite these large inaccuracies, the stable domain is still comparable in size to the stable region of the kinematic FSA controller, and the system response is still significantly faster. The settling time value in Table 2 even improved compared to using accurate model parameters, but that depends on the definition of settling time. If instead of $2 \%$ of $|y(0)|$, a tighter band is used around the equilibrium, it eventually shows that the accurate model converges faster to $y=0$.

The negative effects of the parameter errors become more apparent when comparing the predicted and the real trajectories. Nevertheless, the prediction errors remain small enough to not affect the overall system response much. Therefore if these parameters are not known accurately, overestimating them by a large margin still leads to a fast, highly damped system response that has a clear advantage over the other two controllers. Note that the 
Table 2. Settling time and prediction root mean square error (RMSE) values of numerical simulations.

\begin{tabular}{lccc}
\hline & Settling Time $(\mathrm{s})$ & $\mathrm{RMSE} y(\mathrm{~m})$ & $\mathrm{RMSE} \psi(\mathrm{rad})$ \\
\hline Delayed state feedback & 11.79 & - & - \\
FSA $_{\text {kin }}$ & 9.50 & 0.036 & 0.0019 \\
FSA $_{\text {dyn }}$ accurate model & 4.54 & 0.008 & 0.0021 \\
FSA $_{\text {dyn }}$ overestimated parameters & 4.32 & 0.026 & 0.0042 \\
\hline
\end{tabular}

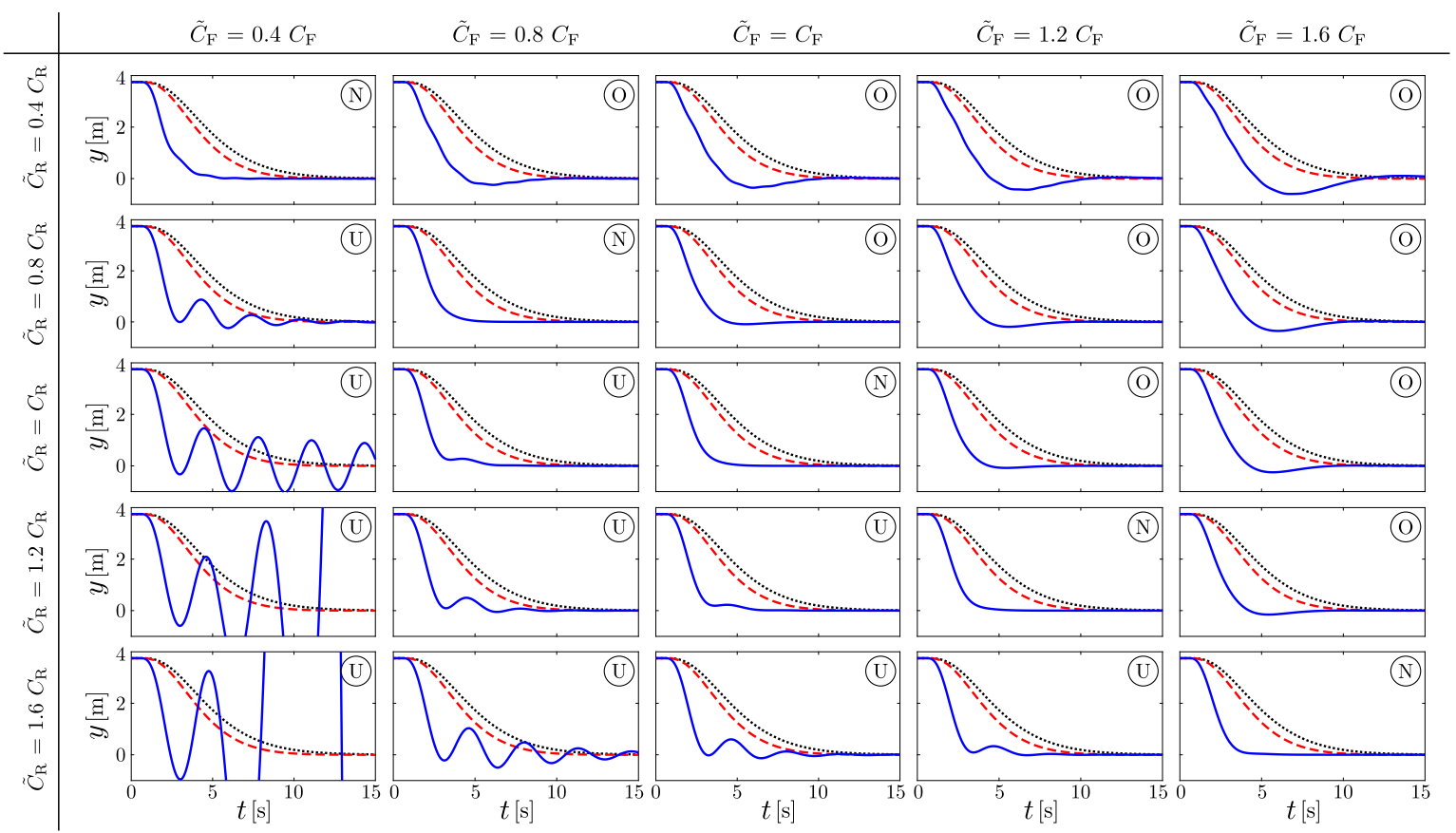

Assumed steering characteristic of the predictive model: (N) neutral, (U) understeer, (O) oversteer ......... Delayed state feedback --- FSA $_{\text {kin }} \quad-$ FSA $_{\text {dyn }}$

Figure 7. Simulation results for $0 \%, \pm 20 \%$ and/or $\pm 60 \%$ inaccuracies in the tire cornering stiffnesses used in the predictive model of the dynamic FSA. Vehicle parameters are listed in Table 1.

control parameters $P_{y}$ and $P_{\psi}$ were not changed, they were set assuming no parameter errors in the predictive model.
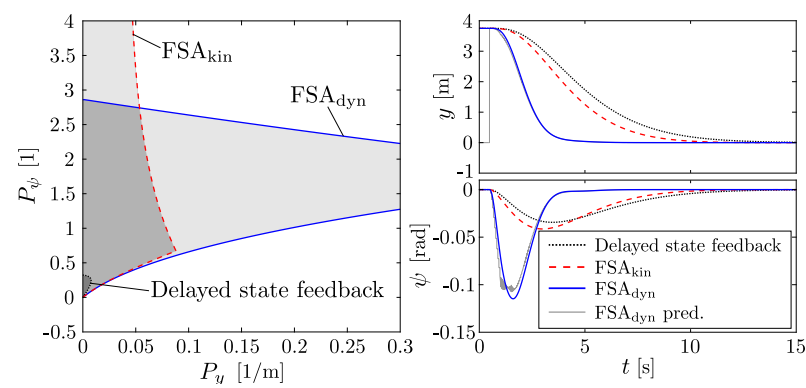

Figure 8. Stability chart and simulation results of a worst case scenario, where the cornering stiffnesses are overestimated by $100 \%$ and the vehicle mass and moment of inertia by $50 \%$ in the predictive model of the dynamic FSA. Vehicle parameters are listed in Table 1 and numerical results in Table 2.

\section{Conclusion}

Due to the growing amount of sensor data and the increasing complexity of the related algorithms (from object detection to vehicle localization), time delay has become a serious issue in autonomous driving. The use of finite spectrum assignment in vehicle motion control can provide a real solution to this problem, directly increasing the safety and efficiency of the related driver assistance and autonomous driving functions. We showed that the presence of feedback delay severely restricts the region of control parameters that can stabilize the vehicle using state feedback. The FSA controller can alleviate this problem by employing a predictive model of the system. Even if this internal model is overly simple with no considerations of tireroad dynamics, the larger admissible control gains already lead to a faster system response. However, extending the predictive model with linear tire characteristics leads to huge improvements both in terms of stable parameter domains and system response, at the cost of approximately four times as many operations. If the increased computational requirements can be handled, then the use of this more detailed model is recommended even if exact values of the additional vehicle parameters are not known: the system's robustness was demonstrated against highly overestimated tire cornering stiffness, vehicle mass and moment of inertia values. As long as the predictive model is not tuned to have an understeering cornering characteristic, the system response is barely affected by variations of these parameters. Although the benefits of the FSA method were demonstrated through the example of a simple feedback controller, similar improvements can be expected in more advanced control approaches too by using the predicted states instead of the delayed signal. 


\section{Declaration of conflicting interests}

The Authors declare that there is no conflict of interest.

\section{Funding}

This research was partly supported by the National Research, Development and Innovation Office under grant no. NKFI-128422 and by the Higher Education Excellence Program of the Ministry of Human Capacities in the frame of Artificial intelligence research area of Budapest University of Technology and Economics (BME FIKP-MI).

\section{References}

Amer NH, Zamzuri H, Hudha K and Kadir ZA (2017) Modelling and control strategies in path tracking control for autonomous ground vehicles: a review of state of the art and challenges. Journal of Intelligent \& Robotic Systems 86(2): 225-254.

Beal CE and Gerdes JC (2012) Model predictive control for vehicle stabilization at the limits of handling. IEEE Transactions on Control Systems Technology 21(4): 1258-1269.

Della Rossa F and Mastinu G (2018) Analysis of the lateral dynamics of a vehicle and driver model running straight ahead. Nonlinear Dynamics 92(1): 97-106.

Elbanhawi M, Simic M and Jazar R (2018) Receding horizon lateral vehicle control for pure pursuit path tracking. Journal of Vibration and Control 24(3): 619-642.

Fierro R and Lewis FL (1997) Control of a nonholomic mobile robot: Backstepping kinematics into dynamics. Journal of robotic systems 14(3): 149-163.

Greenwood DT (2006) Advanced Dynamics. Cambridge University Press.

Heredia G and Ollero A (2007) Stability of autonomous vehicle path tracking with pure delays in the control loop. Advanced Robotics 21(1-2): 23-50.

Hoffmann GM, Tomlin CJ, Montemerlo M and Thrun S (2007) Autonomous automobile trajectory tracking for off-road driving: Controller design, experimental validation and racing. In: American Control Conference, 2007. ACC'07. IEEE, pp. 2296-2301.

Insperger T and Stépán G (2011) Semi-Discretization for TimeDelay Systems. Springer.

Jalali M, Khajepour A, Chen Sk and Litkouhi B (2017) Handling delays in yaw rate control of electric vehicles using model predictive control with experimental verification. Journal of Dynamic Systems, Measurement, and Control 139(12): 121001.

Kyrychko Y and Hogan S (2010) On the use of delay equations in engineering applications. Journal of vibration and control 16(7-8): 943-960.

Liu Q, Liu Y, Liu C, Chen B, Zhang W, Li L and Ji X (2018) Hierarchical lateral control scheme for autonomous vehicle with uneven time delays induced by vision sensors. Sensors 18(8): 2544.

Liu S, Tang J, Zhang Z and Gaudiot JL (2017) Computer architectures for autonomous driving. Computer 50(8): 18-25.

Manitius AZ and Olbrot AW (1979) Finite spectrum assignment problem for systems with delays. IEEE Transactions on Automatic Control 24(4): 541-553.

Mashadi B, Mahmoodi-K M, Kakaee AH and Hosseini R (2013) Vehicle path following control in the presence of driver inputs.
Proceedings of the Institution of Mechanical Engineers, Part K: Journal of Multi-body Dynamics 227(2): 115-132.

Mashadi B, Mahmoudi-Kaleybar M, Ahmadizadeh P and Oveisi A (2014) A path-following driver/vehicle model with optimized lateral dynamic controller. Latin American journal of solids and structures 11(4): 613-630.

Michiels W, Mondié S and Roose D (2003) Robust stabilization of time-delay systems with distributed delay control laws: Necessary and sufficient conditions for a safe implementation. Technical report, Katholieke Universiteit Leuven, Belgium.

Michiels W and Niculescu SI (2007) Stability and Stabilization of Time-Delay Systems. Society for Industrial \& Applied Mathematics, U.S.

Mobus R and Zomotor Z (2005) Constrained optimal control for lateral vehicle guidance. In: IEEE Proceedings. Intelligent Vehicles Symposium, 2005. IEEE, pp. 429-434.

Molnár T and Insperger T (2016) On the robust stabilizability of unstable systems with feedback delay by finite spectrum assignment. Journal of Vibration and Control 22(3): 649-661.

Mondié S, Dambrine M and Santos O (2002) Approximation of control laws with distributed delays: a necessary condition for stability. Kybernetika 38(5): 541-551.

Mondié S and Michiels W (2003) Finite spectrum assignment of unstable time-delay systems with a safe implementation. IEEE Transactions on Automatic Control 48(12): 2207-2212.

Oliveira GL, Burgard W and Brox T (2016) Efficient deep models for monocular road segmentation. In: 2016 IEEE/RSJ International Conference on Intelligent Robots and Systems (IROS). IEEE, pp. 4885-4891.

Olofsson B and Nielsen L (2020) Using crash databases to predict effectiveness of new autonomous vehicle maneuvers for lanedeparture injury reduction. IEEE Transactions on Intelligent Transportation Systems .

Pacejka HB (2006) Tyre and Vehicle Dynamics. ButterworthHeinemann, Oxford.

Paden B, Čáp M, Yong SZ, Yershov D and Frazzoli E (2016) A survey of motion planning and control techniques for selfdriving urban vehicles. IEEE Transactions on intelligent vehicles 1(1): 33-55.

Pendleton S, Andersen H, Du X, Shen X, Meghjani M, Eng Y, Rus D and Ang M (2017) Perception, planning, control, and coordination for autonomous vehicles. Machines 5(1): 6 .

Petrovskaya A and Thrun S (2009) Model based vehicle detection and tracking for autonomous urban driving. Autonomous Robots 26(2-3): 123-139.

Segel L (1956) Theoretical prediction and experimental substantiation of the response of the automobile to steering control. Proceedings of the Institution of Mechanical Engineers: Automobile Division 10(1): 310-330.

Stépán G (1989) Retarded Dynamical Systems: Stability and Characteristic Functions. Longman Scientific \& Technical.

Vörös I and Várszegi B (2018) Lateral vehicle control using finite spectrum assignment. IFAC-PapersOnLine 51(14): 306-311.

Vörös I, Várszegi B and Takács D (2019) Lane Keeping Control Using Finite Spectrum Assignment With Modeling Errors. In: Dynamic Systems and Control Conference (DSCC) 2019. ASME.

Wang QG, Tong HL and Kok KT (1999) Finite-Spectrum Assignment for Time-Delay Systems. Springer-Verlag London. 